\title{
VALIDEZ CURRICULAR DE LAS SERIES ANIMADAS DE TELEVISIÓN PARA LA FORMACIÓN DE FUTUROS MAESTROS: UNA EXPERIENCIA DE TRABAJO
}

\author{
Verónica Marín Díaz \\ Universidad de Córdoba \\ $\mathbf{M}^{\mathbf{a}}$ José Latorre Medina \\ Universidad de Granada
}

\begin{abstract}
RESUMEN
Los medios de comunicación e información de hoy se encuentran inmersos en todas las áreas de desarrollo de nuestra vida, circunstancia que provoca que los poderes, tanto políticos como sociales y educativos, se preocupen de la repercusión que estos manifiestan en nuestros niños y adolescentes. Este artículo presenta los resultados de un estudio realizado en la Universidad de Córdoba, concretamente en la Facultad de Ciencias de la Educación, en el cual se ha tratado de establecer si los programas de televisión, y más concretamente los dibujos animados que muchos niños consumen sin formación previa, podrían ser un recurso para desarrollar determinados contenidos del curriculum de primaria.
\end{abstract}

PALABRAS CLAVE: Medios de comunicación, Televisión, Dibujos animados, Curriculum, Formación de profesores.

\section{ABSTRACT}

Media are actually involved in every aspect of our lives, what makes the political, social and educational forces be worried about their influence in our adolescents and children. This article summarizes the results of a research made by the University of Cordoba (at the Faculty of Education) that has studied the possibility that cartoons and TV programmes can be considered as a resource to develop specific contents of the Primary Education Curriculum.

KEY WORDS: Media, Television, Animate Drawings, Curriculum, Teachers Training. 


\section{INTRODUCCIÓN}

El avance de las tecnologías ha permitido al ser humano desarrollar nuevos soportes y/o formatos para no sólo transmitir ideas, sino también para estar en contacto con otros sujetos o instituciones. Las tecnologías de la imagen y el sonido van cobrando día a día mayor importancia en detrimento de otros sistemas de comunicación que, aunque no han caído en el olvido, en función del estadio generacional en el que nos movamos, quizás sí se han visto relegados a un segundo plano, si bien la comunicación a través de ellos continua produciéndose. Los medios de comunicación e información se configuran hoy día como nuestro medio de existencia, afirma Pérez Tornero (2003, 58); en algo imprescindible que nos permiten construir y desarrollar el conocimiento y las experiencias de enseñanza-aprendizaje necesarias para mejorar nuestra visión del mundo (Marín, 2005a: 225).

La presencia de los medios de comunicación en todas las áreas (política, económica, educativa, familiar...) es algo ya habitual; hemos de aprender así a vivir y convivir con ellos, conocerlos en profundidad pues su capacidad de influir, formar $o$ deformar, promover la cultura o incitar a la marginalidad, informar o desinformar (Pinedo, 1999: 112) van a determinar la mayoría de nuestras relaciones. Estas circunstancias nos hacen plantearnos la necesidad de introducir los medios dentro del ámbito formal de las relaciones del sujeto. Es decir, en virtud del poder que los medios desarrollan sobre nuestras vidas, debemos tratar de controlar su penetración a través de dos ámbitos fundamentalmente: la familia y la escuela, pues son en éstos donde el sujeto pasa la mayor parte de su vida, además de ser su fuente de socialización primaria.

De este modo, partimos de la idea de que la inclusión de los medios en el currículum educativo no implica una modificación de la conducta del sujeto. Consideramos, igualmente, que enseñando al individuo a ver la parte didáctica del medio se puede incluir en el diseño curricular sin ningún tipo de problema. Su integración perseguirá que los alumnos aprendan a desarrollar los sentidos, la inteligencia, la afectividad, la sociabilidad y la eticidad (Rus, 2003: 4).

La educación desarrollada a través de los medios de comunicación tiene que permitir que los niños y adolescentes así como los jóvenes aprendan a discriminar lo que es importante de lo que no lo es de aquella información que les llega, además de saber detectar aquello que es ignorado de forma consciente, todo ello siempre desde una perspectiva crítica y selectiva.

No debemos olvidar que entre las funciones que tienen los medios, además de informar y entretener, está la de vigilancia, ser un foro de debate continuo, educar y transmitir cultura y como no, socializar. Pensamos, por ello, que su integración como recurso curricular es necesaria.

La característica principal de los mass media que puede ayudarnos a incluirlos en determinados puntos o aspectos del currículum escolar es su atractivo y la posibilidad de poder acceder a ellos desde cualquier punto, pudiendo también llegar a cualquier lugar con ellos en cualquier momento.

No obstante, cabe plantearnos si a los medios de comunicación se les puede o no considerar un recurso educativo. Marqués (2001) nos da la respuesta:

- Vehiculan mensajes informativos variados, sugestivos y audiovisuales.

- El conjunto de sus informaciones resulta multicultural. 
- Difunden las noticias de manera inmediata.

- Proporcionan información sobre entornos, personas y fenómenos alejados.

- Despiertan la curiosidad y la imaginación.

- Enseñan de manera práctica el lenguaje audiovisual.

- Constituyen un instrumento de socialización de las personas (modelos de actuación, valores...).

- Difunden la cultura en todos sus aspectos (no es elitista).

- Proporcionan temas comunes de conversación y debate.

- Entretienen.

Y lo deja claro. Estas son razones suficientes para pensar que los medios son o pueden convertirse en una herramienta que ayude a desarrollar el currículum escolar, sin pasar por alto que estos instrumentos también pueden marcar negativamente a los sujetos: según los datos aportados por Bartholow y Anderson (2002), los niños de 12 años han podido ver a lo largo de un año más de cien mil actos violentos en los medios, por lo que la gestión del consumo racional y crítico debe ser uno de los objetivos a cubrir desde los centros escolares.

Desde una perspectiva innovadora, y tratando de superar aspectos negativos como el que acabamos de señalar, hemos de empezar a considerar a los medios como catalizadores de experiencias, como dinamizadores de la comunicación o como objetivos de estudio en sí mismos (Aguaded, 1999: 138).

\section{LA TELEVISIÓN Y SU POSIBILIDAD EDUCATIVA}

La aparición de la televisión en el universo comunicativo y relacional del hombre supuso una revolución de los sistemas económicos, sociales y políticos del momento. Desde su creación en la década de los cuarenta se convirtió en un elemento socializador de la vida de todos los sujetos que entran en contacto, directo o indirecto, con ella, además de mantenerles informados de todo aquello que sucede a su alrededor. Los niños y niñas no contaban con otro modelo que el procedente de la familia o su entorno inmediato, y los medios audiovisuales se convirtieron en unos nuevos modelos mucho más atractivos e impactantes (Rajadell, Pujol y Violent, 2005). La televisión, al ser el más cercano a ellos, se convierte en una fuente de información que no requiere de ninguna habilidad específica para poder acceder a ella, potenciando así la pasividad del televidente. La televisión... deja sin movimiento al ciudadano -esboza Martínez-Salanova (2005)- que queda estático ante la pantalla, le comunica lo que le interesa, le enseña lo que quiere, lo divierte, lo educa, lo duerme, lo acompaña en sus comidas y en su ocio (162).

En estos momentos, la televisión es, junto con internet, el medio de comunicación más fácilmente aceptado por todos y, paradójicamente, el que más odios y pasiones levanta a un mismo tiempo. Su influencia es tal que incluso está presente en todo lo que hacemos, decimos o pensamos. Tal circunstancia pone de manifiesto las tres principales aplicaciones que se la han asignado: teleguardería, teleconsumo y teledivulgación (Feria, 1995). Si visionamos el programa Supernanny emitido la pasada primavera por la cadena Cuatro los vienes por la noche, en torno a las 22:30 horas, podremos ver como su principal objetivo es, a parte de la reeducación de los 
pequeños, el desarrollo de la comunicación entre los diferentes miembros de la familia, evitando que la televisión esté presente en los momentos más importantes del día, como son los desayunos, comidas y cenas. Desde este programa se pretende ganar la batalla a las tres funciones antes mencionadas.

Según Marín (2005b), es hora de superar la concepción negativa sembrada en los últimos años en torno a la televisión. Las funciones citadas anteriormente también desprenden aspectos positivos como son su inmediatez (reduce la distancia espaciotemporal entre el sujeto y el foco de la información), ubicuidad (penetra en el individuo tanto por la vista como por el oído) y brevedad (las imágenes proyectadas tienen un carácter efímero) (Aguaded, 1999).

Todos estos aspectos nos hacen plantearnos la posibilidad de emplear la televisión como una herramienta que nos ayude a desarrollar la educación de los niños y adolescentes. Este medio, tal como afirma Aguaded (2002), está abarcando espacios del saber y la cultura que la escuela había considerado exclusivos (13). Por tanto, puede y debe ser entendido como un elemento más en los procesos de enseñanzaaprendizaje, como un instrumento que facilita la transmisión de la cultura.

A este respecto, Vives (2005) considera que los mass media, concretamente, la televisión, ayudan (a veces de forma semiinconsciente) a los niños y adolescentes a obtener información -de cualquier tipo, educativa o trivial- para poder relacionarse tanto con su grupo de iguales como con la familia.

Por la documentación existente al respecto, sabemos que la televisión y la educación presentan una serie de rasgos característicos que hace que se complementen, como son, entre otros, los siguientes:

\section{Cuadro $n^{\circ}$ 1. Características de la televisión y la educación}

\begin{tabular}{|l|l|}
\hline TELEVISIÓN & EDUCACIÓN \\
\hline - Centrada en el espectáculo y & $\begin{array}{l}\text { - Centrada en la formación. } \\
\text { en el entretenimiento. }\end{array}$ \\
- Pendiente del mundo de hoy & Basada en el rigor. \\
(actualidad). & Su relación con el público es \\
- Su relación con el público es de interés. & - Trabaja desde una perspectiva \\
- Discurso fragmentado. & histórica. \\
- Da la sensación de vivir la realidad. & $\begin{array}{l}\text { - Discurso sosegado y razonado } \\
\text { - Lenguaje de transmisión audiovisual. }\end{array}$ \\
$\begin{array}{l}\text { - Empela la distancia en la expli- } \\
\text { cación de los acontecimientos. }\end{array}$ \\
$\begin{array}{l}\text { - Lenguaje de transmisión escrito } \\
\text { (Aguaded, 2002). }\end{array}$ \\
\hline
\end{tabular}

Sin duda, la combinación de ambos elementos puede ayudar a desarrollar el currículum escolar. Además, el empleo de la televisión en educación presenta una serie de ventajas como son (Cabero, 1994: 164): 
- Lleva a la institución y la educación a lugares apartados o con carencia de profesores especializados.

- Presenta experimentos en aquellos centros con carencia de laboratorios.

- Facilita la introducción en el aula de profesores y personas especializadas en temas concretos.

- Evita desplazamiento de los alumnos a los centros de información y formación.

- Simultáneamente es un medio de comunicación de masas y un medio individual de aprendizaje.

- Ayuda al perfeccionamiento de profesorado....

- Favorece la igualdad de oportunidades para todos los alumnos que observan el programa...

- Facilita una rápida discriminación de las nuevas ideas del currículo y las innovaciones que sobre el mismo se están promoviendo.

- Tiende a mantener actualizados los contenidos de las materias.

- Los contenidos son presentados de forma atractiva para los estudiantes.

Pese a ello, hemos de saber conjugar el fin que cada una de ellas posee -la televisión, entretener y la educación, formar- de cara a la superación de los posibles errores que la integración de la televisión en el diseño curricular pueden provocar: centrarse en el propio medio, falta de estrategias de estructuración y organización de la información, actitudes negativas de los receptores hacia el medio televisivo... (Cabero, 2000), percibirlo como un obstáculo para la autoformación y la creación de una conciencia crítica, además de una identidad cultural, etc.

Una vez superados estos errores o limitaciones, debemos ver la televisión como una fuente de conocimiento y cultura del que, como señalan Sevillano y Perlado (2005), el niño y el adolescente aprenden información, conceptos actitudes, conductas, valores y significados. Debemos combinar la emoción y la curiosidad que provoca la televisión con la cultura, los valores y los modelos que transmite la educación. La conjugación de la televisión y la educación buscará el desarrollo de una conciencia crítica en el consumo de este medio de comunicación.

Los niños y adolescentes son atraídos por la televisión por su lenguaje fácil y rápido, por empleo de imágenes dinámicas y ricas en color. Un claro ejemplo de ello son las series de dibujos animados que podemos visionar en los programas infantiles los fines de semana, $y$, durante la semana, en determinadas cadenas (Antena 3 con los Simpson o Shin Chan, por ejemplo). El estudio realizado por Garitaonadia, Juarista y Oleada (1999) señala como el $66 \%$ de los niños con edades comprendidas entre los 6 y 7 años prefieren ver dibujos animados. Podemos comprobar con sólo asomarnos a las pantallas de nuestros receptores televisivos que los programas destinados al público infantil y juvenil se nutren de series de ficción protagonizadas por personajes tanto de carne y hueso como animados que atraen totalmente su atención.

La fantasía con la que están ideadas este tipo de series, a veces, pueden hacer que la línea que separa la ficción de la realidad desaparezca haciendo que el público adulto las considere perniciosas (Marín, 2005b). Pensamos que es ahí donde tanto la familia como la escuela deben intervenir. Como ya señalamos antes, no debemos considerar toda la programación televisiva como algo negativo; también hay progra- 
mas ('El club de las ideas' de Canal 2 Andalucía, por ejemplo) así como algunas series de dibujos animados que posen una alta calidad, además de transmitir valores tanto didácticos como culturales.

En definitiva, los centros escolares deben apostar por la enseñanza de un consumo racional de los mass media, en este caso, de la televisión, entrenando a los más pequeños para ver de forma crítica este medio.

\section{DESARROLLO DE LA EXPERIENCIA DE TRABAJO}

La utilización de la televisión en los centros educativos como recurso para desarrollar el currículum debería ser una realidad. Por un lado, sabemos que muchos profesores no lo emplean por falta de una formación adecuada para hacerlo, y, por otro, porque no le otorgan validez suficiente, considerando que no es un recurso adecuado para la puesta en marcha del currículum. De este modo, pensamos que desde la formación universitaria que reciben los futuros maestros se debe potenciar su empleo.

El objetivo del presente estudio consistía en determinar el grado de validez curricular que las series de dibujos animados proyectadas en los programas de corte infantil tenían, en función de si éstas cumplían los objetivos curriculares establecidos en la legislación vigente para la etapa, en este caso, de Primaria. Para ello, reelaboramos y reenunciamos los objetivos para esta etapa en la LOCE y la LOGSE, dada la situación política que se vivía en ese momento con respecto a la aplicación de la normativa.

Entre todas las series animadas que las diferentes cadenas de televisión proyectaban, escogimos al azar "La Banda del Patio", que llevaba proyectándose en nuestras pantallas más de cinco años, pasando por diferentes cadenas. En aquél momento se ubicaba dentro del programa "Club Disney" que en la actualidad se ofrece en la Primera de televisión española.

"La Banda del Patio" es una serie protagonizada por un grupo de amigos y compañeros donde cada uno tiene un rol característico. En esta pandilla encontramos al líder en la figura de TJ, que personifica a un niño atrevido y osado capaz de ver lo bueno y lo malo tanto de las personas como de las cosas, somete las decisiones a votación por el grupo de amigos y es el que se encuentra más integrado en el resto de compañeros del patio del recreo. Spinelly es una niña que representa la fuerza, la bravuconería, conducta poco propia en una niña. Su contrapunto lo pone Miky, un niño grande y con sobrepeso al que le gusta la poesía y trata sólo de ver la bondad de todas las cosas y personas. La pasión por el deporte y el afán de superación y de ser siempre el número uno está reflejado en Bin. Gus representa al niño nuevo, con todas sus inseguridades y dificultades para ser aceptado por los demás y, por último, encontramos a Grechel, la más lista del colegio. También existen personajes secundarios como Rey Bob, un alumno de cursos superiores encargado de gobernar el recreo, que representa el poder y la equidad o Randal, quien personifica al chivato del patio del recreo.

Una vez facilitada esta información sobre los protagonistas, un grupo de futuros maestros de la Universidad de Córdoba -que conformaron la muestra del estudio, como explicamos a continuación- visionaron dos episodios de la serie con una duración aproximada de 16 minutos cada uno de ellos. En el primero, sus protagonistas daban una lección de amistad y compañerismo al resto de sus compañeros de aula 
y de recreo, pues a uno de ellos se le rompen los pantalones, circunstancia que hace que sea humillado públicamente ante todo el alumnado del recreo por parte de la profesora encargada de vigilarlo. En el segundo capítulo, la llegada de un estudiante nuevo provoca, primero, una sensación de rechazo, ya que es un alumno superior a todos ellos en todos los campos, materias y actividades, para después ser aceptado por todos los compañeros.

\section{Población y muestra de investigación}

La población objeto de estudio la conformaban estudiantes que estaban cursando la asignatura 'Nuevas Tecnologías Aplicadas a la Educación' en el primer curso de la Titulación de Maestro en las especialidades de Lengua Extranjera y Educación Física en la Universidad de Córdoba durante el curso académico pasado. La muestra de investigación estuvo formada por todos los aprendices de profesor que asistieron a clase el día en el cual se desarrolló esta experiencia de trabajo. En total, son 124 futuros maestros los que participaron en este estudio.

\section{Descripción de la muestra}

De los 124 estudiantes que constituyeron la muestra, 69 son estudiantes de Lengua Extranjera y 55 de Educación Física. Son mayoría las mujeres (59,7\%) que los hombres (40,3\%). Más del 80,0\% no superan los 23 años de edad (83,9\%). En los gráficos siguientes podemos visualizar mejor la distribución de los sujetos del estudio según el sexo y la edad.

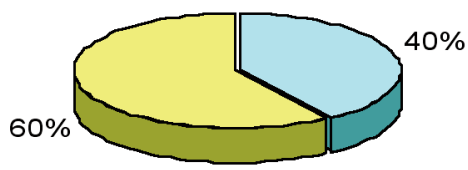

\section{Hombres $\square$ Mujeres}

Gráfico nº 1. Distribución de la muestra por sexo

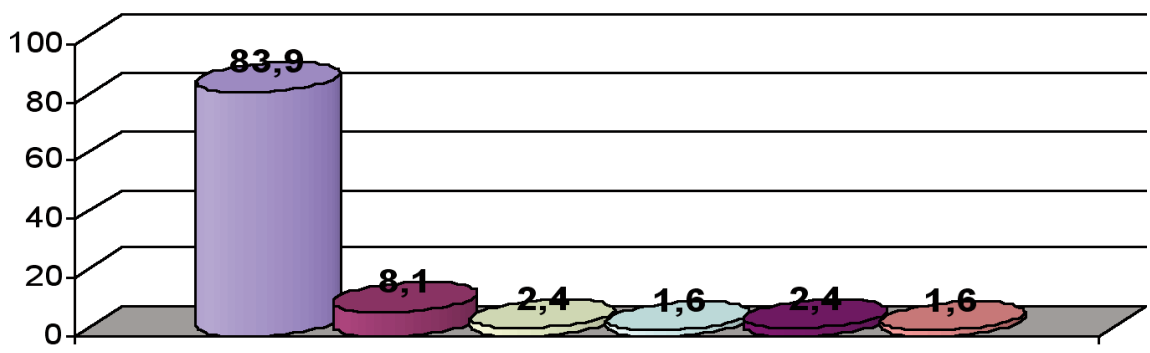

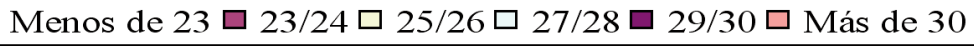

Gráfico $n^{\circ} 2$. Distribución de la muestra por edad 


\section{Recogida y análisis de los datos}

Para la recogida de los datos, empleamos el cuestionario como técnica de investigación cuantitativa. El cuestionario estaba formado inicialmente por 25 ítems en un formato de escala tipo Likert con cinco alternativas de respuesta, donde el valor 1 equivalía a 'No se cumple totalmente' y el 5, 'Se cumple totalmente'. Una vez construido el instrumento lo sometimos a validez de contenido por medio de un grupo de jueces externos especialistas en la materia, quedando finalmente compuesto por 21 ítems.

El tratamiento estadístico aplicado a los datos recogidos mediante el cuestionario ha sido el siguiente: en primer lugar, hemos realizado un análisis descriptivo. Los estadísticos descriptivos básicos: media aritmética, desviación típica y distribución de frecuencias que han sido calculados para cada una de las variables nos han permitido, además de obtener una visión global de los sujetos que integran la muestra, aproximarnos lo mejor posible a las opiniones emitidas por los mismos.

$\mathrm{Y}$, en segundo lugar, hemos llevado a cabo un análisis factorial. En concreto, hemos realizado una extracción de componentes principales y una rotación ortogonal, la rotación Varimax de Kaiser (Etxeberria y Tejedor, 2005).

Para realizar ambos tipos de análisis, recurrimos al paquete estadístico de ordenador SPSS, versión 12.01 para el entorno Windows.

\section{RESULTADOS}

\subsection{Del análisis descriptivo}

Los datos correspondientes a los porcentajes, medias y desviaciones típicas obtenidos se recogen en la tabla ${ }^{0} 1$.

Una rápida mirada por las evidencias anteriores nos pone en conocimiento de que los ítems mejor valorados, según las opiniones esbozadas por los futuros maestros encuestados, dicen relación con el fomento del empleo del deporte como medio para el desarrollo personal y social, con el desarrollo de una actitud responsable y de respeto hacia los demás, con favorecer la creación de un clima propicio para la libertad personal, el aprendizaje y la convivencia, con permitir conocer los valores y normas de convivencia, con fomentar el respeto a la pluralidad del centro, con el aprecio por la naturaleza y el entorno, así como con la oportunidad para iniciarse en el conocimiento de la higiene y la salud.

En contrapartida, encontramos que las declaraciones peor valoradas por los aprendices de profesor aluden a la ayuda proporcionada para conocer los aspectos fundamentales de la Geografía, por un lado, y los de Historia y Cultura, por otro; a la ayuda para desarrollar hábitos de lectura y al apoyo para iniciarse en la resolución de problemas que requieren la realización de operaciones de cálculo, conocimientos geométricos y estimaciones.

\subsection{Del análisis factorial}

Los resultados del análisis factorial realizado ponen de manifiesto la existencia de cinco factores. En la tabla siguiente recogemos el porcentaje de la varianza explicada por cada uno de los factores resultantes, junto con la proporción acumulativa de la varianza explicada. El total de la varianza explicada por los cinco conglomerados considerados es igual a $68,945 \%$. 
Tabla $\mathbf{n}^{\circ}$ 1. Datos correspondientes a las valoraciones efectuadas por los futuros maestros

\begin{tabular}{|c|c|c|c|c|c|c|c|}
\hline \multirow[t]{3}{*}{ ÍTEMS DEL CUESTIONARIO } & \multicolumn{7}{|c|}{ Futuros Docentes } \\
\hline & \multicolumn{5}{|c|}{ Porcentajes } & \multirow[t]{2}{*}{ MED. } & \multirow[t]{2}{*}{ D.T. } \\
\hline & 1 & 2 & 3 & 4 & 5 & & \\
\hline 1. Permite conocer los valores y normas de convivencia & 1,6 & 4,9 & 29,3 & 46,3 & 17,9 & 3,74 & 0,867 \\
\hline 2. Fomenta el respeto a la pluralidad del centro & 0,8 & 10,5 & 33,1 & 33,1 & 22,6 & 3,66 & 0,970 \\
\hline $\begin{array}{l}\text { 3. Ayuda a desarrollar hábitos de esfuerzo y responsabilidad } \\
\text { en el estudio }\end{array}$ & 3,3 & 16,3 & 36,6 & 37,4 & 6,5 & 3,28 & 0,926 \\
\hline 4. Ayuda a desarrollar la curiosidad por el aprendizaje & 4,1 & 20,3 & 35,8 & 23,6 & 16,3 & 3,28 & 1,089 \\
\hline $\begin{array}{l}\text { 5. Desarrolla una actitud responsable y de respeto hacia } \\
\text { los demás }\end{array}$ & 1,6 & 4,9 & 26,8 & 34,1 & 32,5 & 3,91 & 0,967 \\
\hline $\begin{array}{l}\text { 6. Favorece la creación de un clima propicio para la } \\
\text { libertad personal, el aprendizaje y la convivencia }\end{array}$ & 2,4 & 8,9 & 25,0 & 33,9 & 29,8 & 3,80 & 1,044 \\
\hline 7. Desarrolla la iniciativa individual & 2,4 & 18,5 & 25,0 & 34,7 & 19,4 & 3,50 & 1,078 \\
\hline 8. Ayuda a conocer la lengua castellana & 8,1 & 22,8 & 34,1 & 21,1 & 13,8 & 3,10 & 1,148 \\
\hline 9. Ayuda a desarrollar hábitos de lectura & 13,8 & 28,5 & 37,4 & 13,8 & 6,5 & 2,71 & 1,077 \\
\hline $\begin{array}{l}\text { 10. Ayuda en la iniciación de la resolución de problemas } \\
\text { que requieren la realización de operaciones de cálculo, } \\
\text { conocimientos geométricos y estimaciones }\end{array}$ & 13,8 & 30,1 & 29,3 & 18,7 & 8,1 & 2,77 & 1,151 \\
\hline $\begin{array}{l}\text { 11. Ayuda a conocer los aspectos fundamentales de las } \\
\text { Ciencias de la Naturaleza }\end{array}$ & 7,3 & 21,0 & 41,1 & 25,0 & 5,6 & 3,01 & 0,992 \\
\hline $\begin{array}{l}\text { 12. Ayuda a conocer los aspectos fundamentales de } \\
\text { la Ceografía }\end{array}$ & 17,1 & 35,8 & 33,3 & 11,4 & 2,4 & 2,46 & 0,986 \\
\hline $\begin{array}{l}\text { 13. Ayuda a conocer los aspectos fundamentales de la } \\
\text { Historia y la Cultura }\end{array}$ & 17,9 & 35,0 & 28,5 & 15,4 & 3,3 & 2,51 & 1,059 \\
\hline $\begin{array}{l}\text { 14. Inicia al sujeto en el aprendizaje de las Tecnologías de } \\
\text { la Información y Comunicación }\end{array}$ & 4,9 & 26,0 & 42,3 & 21,1 & 5,7 & 2,97 & 0,949 \\
\hline $\begin{array}{l}\text { 15. Inicia al sujeto en la valoración de diferentes } \\
\text { manifestaciones artísticas }\end{array}$ & 2,4 & 14,5 & 41,9 & 29,0 & 12,1 & 3,34 & 0,953 \\
\hline $\begin{array}{l}\text { 16. Inicia al sujeto en la producción estética de diferentes } \\
\text { manifestaciones artísticas }\end{array}$ & 1,6 & 22,6 & 37,9 & 31,5 & 6,5 & 3,19 & 0,914 \\
\hline 17. Ayuda a conocer el valor del propio cuerpo & 4,8 & 10,5 & 27,4 & 36,3 & 21,0 & 3,58 & 1,083 \\
\hline 18. Inicia en el conocimiento de la higiene y la salud & 3,3 & 9,8 & 27,6 & 39,0 & 20,3 & 3,63 & 1,018 \\
\hline $\begin{array}{l}\text { 19. Fomenta el empleo del deporte como medio para el } \\
\text { desarrollo personal y social }\end{array}$ & 0,8 & 4,0 & 23,4 & 41,1 & 30,6 & 3,97 & 0,883 \\
\hline 20. Ayuda a conocer la naturaleza y su entorno & 4,8 & 8,1 & 34,7 & 34,7 & 17,7 & 3,52 & 1,032 \\
\hline 21. Ayuda a valorar la naturaleza y su entorno & 3,3 & 7,4 & 32,8 & 33,6 & 23,0 & 3,66 & 1,019 \\
\hline
\end{tabular}


Tabla $\mathbf{n}^{0}$ 2. Proporción y porcentaje acumulativo de la varianza explicada

\begin{tabular}{|c|c|c|}
\hline FACTORES & $\begin{array}{c}\text { Porcentaje de varianza } \\
\text { explicada }\end{array}$ & $\begin{array}{c}\text { Porcentaje acumulativo } \\
\text { de la varianza }\end{array}$ \\
\hline I & 17,528 & 17,528 \\
\hline II & 15,906 & 33,434 \\
\hline III & 15,829 & 49,263 \\
\hline IV & 11,433 & 60,697 \\
\hline V & 8,249 & 68,945 \\
\hline
\end{tabular}

Tras establecer los ítems componentes de cada factor, describimos cada uno de ellos, indicando el grado de saturación que cada ítem ha alcanzado dentro de cada uno de los conglomerados. Hemos de señalar que se han considerado únicamente aquellos ítems cuyas saturaciones son iguales o superiores a 0,40. Los factores obtenidos han sido nominados de la siguiente forma:

\section{Tabla $\mathbf{n}^{\circ}$ 3. Factor I: "DESARROLLO DE LA CREATIVIDAD"}

\begin{tabular}{|c|l|c|}
\hline ITEM & \multicolumn{1}{|c|}{ DEFINICIÓN } & SATURACIÓN \\
\hline 9 & Ayuda a desarrollar hábitos de lectura & 0,654 \\
\hline 10 & $\begin{array}{l}\text { Ayuda en la iniciación de la resolución de problemas } \\
\text { que requieren la realización de operaciones de } \\
\text { cálculo, conocimientos geométricos y estimaciones }\end{array}$ & 0,536 \\
\hline 11 & $\begin{array}{l}\text { Ayuda a conocer los aspectos fundamentales de } \\
\text { las Ciencias de la Naturaleza }\end{array}$ & 0,692 \\
\hline 12 & $\begin{array}{l}\text { Ayuda a conocer los aspectos fundamentales de la } \\
\text { Geografía }\end{array}$ & 0,859 \\
\hline 13 & $\begin{array}{l}\text { Ayuda a conocer los aspectos fundamentales de la } \\
\text { Historia y la Cultura }\end{array}$ & 0,835 \\
\hline 14 & $\begin{array}{l}\text { Inicia al sujeto en el aprendizaje de las Tecnologías } \\
\text { de la Información y Comunicación }\end{array}$ & 0,583 \\
\hline
\end{tabular}

El primer factor está conformado por dos bloques de variables. Por un lado, las número 11, 12 y 13 dan cuenta de cómo este recurso puede despertar la curiosidad del alumno de educación primaria por conocer aspectos formales del currículum referidos a Geografía, Historia y Cultura y Ciencias de la Naturaleza. Por otro lado, las variables 9,10 y 14 informan de una serie de destrezas y hábitos de aprendizaje que contribuyen al desarrollo de la creatividad en los más pequeños. En consecuencia, nominamos al factor 'Desarrollo de la creatividad'. 
Tabla no 4. Factor II: "EDUCACIÓN PARA LA CIUDADANÍA"

\begin{tabular}{|c|l|c|}
\hline ITEM & \multicolumn{1}{|c|}{ DEFINICIÓN } & SATURACIÓN \\
\hline 1 & Permite conocer los valores y normas de convivencia & 0,778 \\
\hline 2 & Fomenta el respeto a la pluralidad del centro & 0,748 \\
\hline 5 & $\begin{array}{l}\text { Desarrolla una actitud responsable y de respeto hacia } \\
\text { los demás }\end{array}$ & 0,839 \\
\hline 6 & $\begin{array}{l}\text { Favorece la creación de un clima propicio para la } \\
\text { libertad personal, el aprendizaje y la convivencia }\end{array}$ & 0,704 \\
\hline 19 & $\begin{array}{l}\text { Fomenta el empleo del deporte como medio para el } \\
\text { desarrollo personal y social }\end{array}$ & 0,462 \\
\hline
\end{tabular}

Titulamos a este segundo factor 'Educación para la ciudadanía' por cuanto en él se concentran aquellas variables que refieren una serie de valores y actitudes cívicos como son, entre otros, el respeto, tanto hacia la diversidad de los compañeros como al propio centro, la convivencia, el desarrollo de una actitud responsable hacia los demás y hacia uno mismo, que son temas plausibles de desarrollarse a través de la inclusión de esta serie animada en el currículum de primaria.

\begin{tabular}{|c|l|c|}
\hline ITEM & \multicolumn{1}{|c|}{ DEFINICIÓN } & SATURACIÓN \\
\hline 7 & Desarrolla la iniciativa individual & 0,411 \\
\hline 17 & Ayuda a conocer el valor del propio cuerpo & 0,739 \\
\hline 18 & Inicia en el conocimiento de la higiene y la salud & 0,712 \\
\hline 19 & $\begin{array}{l}\text { Fomenta el empleo del deporte como medio para el } \\
\text { desarrollo personal y social }\end{array}$ & 0,563 \\
\hline 20 & Ayuda a conocer la naturaleza y su entorno & 0,764 \\
\hline 21 & Ayuda a valorar la naturaleza y su entorno & 0,727 \\
\hline
\end{tabular}

\section{Tabla no 5. Factor III: "EDUCACIÓN PARA LA SALUD"}

Integran este tercer factor un conjunto de variables que ponen de manifiesto la utilidad de la serie animada de televisión para aprender y fomentar una cultura de la salud en las aulas de educación primaria (conocer y valorar el propio cuerpo y la naturaleza, concebir el deporte como una práctica saludable para el desarrollo personal y social, etc.). Es por ello que le denominamos 'Educación para la salud'. 
Tabla n 6. Factor IV: "DESARROLLO DE HABILIDADES DE APRENDIZAJE"

\begin{tabular}{|c|l|c|}
\hline ITEM & \multicolumn{1}{|c|}{ DEFINICIÓN } & SATURACIÓN \\
\hline 3 & $\begin{array}{l}\text { Ayuda a desarrollar hábitos de esfuerzo y } \\
\text { responsabilidad en el estudio }\end{array}$ & 0,733 \\
\hline 4 & Ayuda a desarrollar la curiosidad por el aprendizaje & 0,617 \\
\hline 7 & Desarrolla la iniciativa individual & 0,575 \\
\hline 8 & Ayuda a conocer la lengua castellana & 0,430 \\
\hline 10 & $\begin{array}{l}\text { Ayuda en la iniciación de la resolución de problemas } \\
\text { que requieren la realización de operaciones de } \\
\text { cálculo, conocimientos geométricos y estimaciones }\end{array}$ & 0,439 \\
\hline 16 & $\begin{array}{l}\text { Inicia al sujeto en la producción estética de } \\
\text { diferentes manifestaciones artísticas }\end{array}$ & 0,429 \\
\hline
\end{tabular}

Hablamos de 'Desarrollo de habilidades de aprendizaje' por cuanto las variables que componen el factor relatan algunos de los requisitos que son clave y necesarios para que el alumno aprenda (iniciativa personal, curiosidad por aprender, esfuerzo y responsabilidad por el estudio, conocimiento de la lengua castellana, vocabulario...) y a cuyo desarrollo parece contribuir efectivamente la utilización de esta serie de dibujos animados.

Tabla n" 7. Factor V: "EDUCACIÓN ARTÍSTICA"

\begin{tabular}{|c|l|c|}
\hline ITEM & \multicolumn{1}{|c|}{ DEFINICIÓN } & SATURACIÓN \\
\hline 15 & $\begin{array}{l}\text { Inicia al sujeto en la valoración de diferentes } \\
\text { manifestaciones artísticas }\end{array}$ & 0,783 \\
\hline 16 & $\begin{array}{l}\text { Inicia al sujeto en la producción estética de } \\
\text { diferentes manifestaciones artísticas }\end{array}$ & 0,742 \\
\hline
\end{tabular}

Este quinto factor recoge dos variables que nos revelan, finalmente, la utilidad de esta serie animada para introducir al alumno en el mundo del arte. Por ello lo titulamos con el nombre 'Educación artística'.

\section{CONCLUSIONES}

Del presente trabajo hemos extraído las siguientes conclusiones:

1. Los ítems mejor considerados por los estudiantes de profesorado están encaminados a la consecución de la mejora personal del sujeto, estrechamente relacionado con su educación cívica y moral, así como la educación en valores y para la salud, todos ellos temas tratados en la legislación anterior (LOGSE), dentro del marco llamado temas transversales. 
2. Los resultados del análisis factorial ponen de manifiesto la existencia de cinco factores que explican el 68,9\% de la varianza total. De este modo, los 21 ítems que conforman el cuestionario han sido reducidas a cinco conglomerados que nos facilitan la comprensión de las opiniones emitidas por los aprendices de profesor.

3. Los factores resultantes han sido nominados en función de las variables que los conforman y nos ayudan a explicarlos: Factor I: 'Desarrollo de la creatividad'; Factor II: 'Educación para la ciudadanía'; Factor III: 'Educación para la salud; Factor IV: 'Desarrollo de habilidades de aprendizaje' y Factor V: 'Educación artística'.

\section{REFLEXIÓN FINAL}

Que en nuestra cultura existe una predisposición a asociar el aparato de televisión con un instrumento dedicado a la diversión más que con un artilugio destinado al trabajo intelectual o profesional es algo incuestionable (Franquet, 1999). Prueba de ello es el consumo masivo que de éste y otros medios de comunicación hace la población infantil y juvenil. Una realidad que reclama la intervención de las familias y los centros educativos para que este consumo se realice de forma crítica. Dado que la intencionalidad comunicativa de los medios va más allá del momento en que se visualizan, su integración en los sistemas educativos cara a la preparación de futuros formadores en el uso de los mismos se erige como una necesidad imperiosa.

Sea como fuere, lo que está claro, por lo expuesto anteriormente, es la validez curricular que series animadas de televisión, como la trabajada en esta experiencia formativa, tienen para el entrenamiento universitario de futuros profesores de primaria. Confiamos, por tanto, en que docentes y profesionales en formación las incorporen como un recurso más a su maleta pedagógica y las utilicen sabiamente para poner en marcha sus programaciones de aula.

\section{BIBLIOGRAFÍA}

Aguaded, J. I. (1999). Convivir con la televisión. Familia, educación y recepción televisiva. Barcelona: Paidos.

Aguaded, J. I. (2002). Aulas en la pantalla. Presente y futuro de la televisión educativa. Huelva: Grupo Comunicar.

Ballesta, J. (2002). Educar para la comunicación masiva: un reto en la formación del ciudadano. Disponible en http://dewey.uab.es/pmarques/evte/ballesta2.doc. Consultado el 20/11/2003.

Bartholow, B. D y Anderson, C.A. (2002). Effects of violent videogames on aggressive behaviour: potential sex differences. Journal of Experimental Social Psychology, 38 (3). 283-290.

Cabero, J. (1994). Retomando un medio: la televisión educativa. CMIDE del Excmo. Ayuntamiento de Sevilla y SAV de la Universidad de Sevilla. Medios de comunicación, recursos y materiales para la mejora educativa. Sevilla: CMIDE del Excmo. Ayuntamiento de Sevilla y SAV de la Universidad de Sevilla. 161-193.

Cabero, J. (2000). La televisión educativa: aspectos a contemplar para su integración curricular. Disponible en http://tecnologíaedu.es/revistaslibros/nov01/tve.htm. Consultado el 16/02/2002. 
Etxeberria, J. y Tejedor, F. J. (2005). Análisis descriptivo de datos en educación. Madrid: La Muralla.

Feria, A. (1995). Educación y televisión. En Actas de las II Jornadas de Comunicación Social. Sevilla: Junta de Andalucía. 8-9.

Franquet, R. (1999). Comunicar en la sociedad de la información. Zer, Revista de Estudios de Comunicación, 7. Disponible en http://www.ehu.es/zer/zer7/ranquet65.htm. Consultado el 14/04/2004.

Garitaonadia, C.; Juarista, P y Oleada, J. (1999). Qué ven y cómo juegan los niños españoles. El uso que los niños y los jóvenes hacen de los medios de comunicación. Zer, Revista de Estudios de Comunicación, 6. Disponible en http://www.ehu.es/zer/zer6/4garito.htm. Consultado el 10/09/2004.

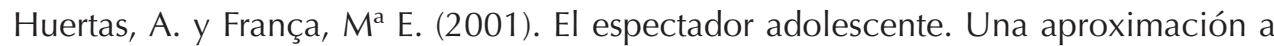
cómo contribuye la televisión a la constitución del yo. Zer, Revista de Estudios de Comunicación, 11. Disponible en http://www.ehu.es/zer/zer11web/huertas.htm. Consultado el 10/09/2004.

Marín, V. (2005a). La televisión de calidad y la familia. Comunicar, 25. 225-230.

Marín, V. (2005b). Las series animadas de televisión y su valor educativo. Actas del Congreso Hispanoluso de Comunicación y Educación "Hacia una TV de calidad. La televisión que queremos...". Huelva: Grupo Comunicar. Libro electrónico.

Martínez-Salanova, E. (2005). Ciudadanos y pantallas: pasividad o responsabilidad. Comunicar, 25.161-166.

Marqués, P. (2001). Aportaciones de los mass media en educación. Problemáticas asociadas. Disponible en http://dewey.uab.es/pmarques/masme.htm. Consultado el $26 / 10 / 2004$.

Pérez Tornero, J. M. (2003). Educación en medios: perspectivas y estrategias. En J. I. Aguaded. Luces en el laberinto audiovisual. Huelva: Grupo Agora Digit@l, Grupo Comunicar y Universidad de Huelva. 57-59.

Pinedo, C. (1999). Televisión y formación. Comunicar, 13. 111-116.

Rajadell, N.; Pujol, Ma A. y Violant, V (2005). Los dibujos animados como recurso de transmisión de los valores educativos y culturales. Actas del Congreso Hispanoluso de Comunicación y Educación "Hacia una TV de calidad. La televisión que queremos...". Huelva: Grupo Comunicar. Libro electrónico.

Rus, A. J. (2003). Educar para la comunicación. Profesorado, Revista de Currículum y Formación del Profesorado, 7 (1/2). 1-5.

Sevillano, $M^{\mathrm{a}}$ L. y Perlado, L. (2005). Los programas televisivos infantiles preferidos por los niños de 6 a 8 años. Actas del Congreso Hispanoluso de Comunicación y Educación "Hacia una TV de calidad. La televisión que queremos...". Huelva: Grupo Comunicar. Libro electrónico.

Vives, M. (2005). La educación del futuro: cambios en la intimidad de los jóvenes. Comunicación y Pedagogía, 207. 53-56. 